\title{
Remote Monitoring and Management of High-Risk Patients Being Started on Antineoplastic Treatment
}

Gilad Kuperman", MD, PhD; Abigail Baldwin-Medsker ${ }^{*}$, RN, MSN, ONC; Margarita Rozenshteyn *; Melissa Zablocki*, MPH; Claire Perry ; Amandeep Dhami ${ }^{*}$, MPH; Brett Simon*, MD, PhD; Robert Daly ${ }^{*}$, MD, MBA; Yeneat Ophelia Chiu ${ }^{*}$, MHA

Memorial Sloan Kettering Cancer Center, New York, United Kingdom

*all authors contributed equally

Corresponding Author:

Gilad Kuperman, MD, PhD

Memorial Sloan Kettering Cancer Center

1275 York Avenue

New York

United Kingdom

Phone: 6464491975

Email: kupermag@mskcc.org

\section{Abstract}

Background: Suboptimal management of cancer-related symptoms can lead to potentially preventable emergency department visits. Early detection and management of these symptoms leads to improved patient outcomes. The goal of this program is to capture symptom data on a daily cadence from patients beginning started on antineoplastic treatment and make these data available to staff who would intervene with the aim of mitigating symptom escalation. The aims of this pilot were patient acceptance of automated questionnaires, use of data by clinical staff, and integration of digital tools into workflows.

Objective: The objective was development and initial evaluation of a digitally enabled program to monitor and manage symptoms of cancer patients being started on treatment with antineoplastic drugs.

Methods: Memorial Sloan Kettering Cancer Center (MSKCC) patients being started on antineoplastic treatment were eligible for inclusion. The technology supporting the program included: (i) a predictive model identifying patients at high risk for emergency department visit in the next 6 months, (ii) a patient portal enabling symptom questionnaires to be sent to enrolled patients, (iii) an internally developed application that allows staff to review and trend symptom data, (iv) "alerts" for concerning symptoms that are sent to a dedicated team of oncology registered nurses and nurse practitioners, and (v) a secure messaging platform for communication between staff and patients. The predictive model runs nightly to identify eligible patients. Physicians review the risk information and decide whether to enroll each patient. Enrolled patients receive a daily patient-reported outcome questionnaire to capture symptoms. Alerts are generated based on the patient's response to the symptom questions. The team reviews symptom data and interacts with the patient via phone, secure messaging, or televisits. The team collaborates with the primary oncology team as appropriate.

Results: The program went live in October 2018. Fifteen medical oncologists are participating in the pilot. As of June 21, 2019, 302 patients have been evaluated by the predictive model, of which 53 have been high risk and 249 have been low risk. Physicians have enrolled a total of 86 patients. Enrolled patients have completed 4198 out of 7287 symptom questionnaires, for a completion rate of 57.6\%. Of the 4198 questionnaires, 1638 have generated a "red alert" (severe) symptom. Over 1200 secure messages have been exchanged between patients and staff. Lessons learned include: (i) patient acceptance of daily questionnaires has been high, (ii) the use of alerts assists the team in proactively managing participating patients, and (iii) patients and caregivers are reporting that they find the program valuable. The pilot demonstrated that daily symptom monitoring through a technology-enabled platform and a dedicated team is feasible and we are examining how the program might be scaled across our organization.

Conclusions: Through the use of automated symptom questionnaires and various bidirectional communication channels, remote monitoring and management of symptoms in cancer patients is feasible. This program enables providers at MSKCC to have visibility into patient symptomatology between visits and improves our understanding of the patient's needs. The workflows of the staff monitoring the symptoms and how best to coordinate symptom management across multiple providers are areas of ongoing refinement. 
(iproc 2019;5(1):e15181) doi: $10.2196 / 15181$

\section{KEYWORDS}

cancer

Edited by R Palacholla; this is a non-peer-reviewed article. Submitted 25.06.19; accepted 14.08.19; published 02.10.19.

Please cite as:

Kuperman G, Baldwin-Medsker A, Rozenshteyn M, Zablocki M, Perry C, Dhami A, Simon B, Daly R, Chiu YO

Remote Monitoring and Management of High-Risk Patients Being Started on Antineoplastic Treatment

iproc 2019;5(1):e15181

URL: http://www.iproc.org/2019/1/e15181/

doi: $10.2196 / 15181$

PMID:

(C) Gilad Kuperman, Abigail Baldwin-Medsker, Margarita Rozenshteyn, Melissa Zablocki, Claire Perry, Amandeep Dhami, Brett Simon, Robert Daly, Yeneat Ophelia Chiu. Originally published in Iproceedings (http://www.iproc.org), 02.10.2019 This is an open-access article distributed under the terms of the Creative Commons Attribution License (https://creativecommons.org/licenses/by/4.0/), which permits unrestricted use, distribution, and reproduction in any medium, provided the original work, first published in Iproceedings, is properly cited. The complete bibliographic information, a link to the original publication on http://www.iproc.org/, as well as this copyright and license information must be included. 